\title{
Research on Evacuation Based on Social Force Model
}

\author{
Liu Wen ${ }^{\text {a }}$, Deng Zhongliang ${ }^{\text {a }}$, Li Wenyi ${ }^{\text {a }}$, Lin Jie ${ }^{\text {a }}$ \\ a Beijing University of Posts and Telecommunications, Beijing, China - buptbehan@126.com, (dengzl, \\ liwenyi)@bupt.edu.cn,595833506@qq.com
}

KEY WORDS: Social Force, Indoor Environment, Evacuation, Disorder, Speed-separation

\begin{abstract}
:
Crowded centers always cause personnel casualties in evacuation operations. Stampede events often occur by hit, squeeze and crush due to panic. It is of vital important to alleviate such situation. With the deepening of personnel evacuation research, more and more researchers are committed to study individual behaviors and self-organization phenomenon in evacuation process. The study mainly includes: 1 , enrich the social force model from different facets such as visual, psychological, external force to descript more realistic evacuation; 2, research on causes and effects of self - organization phenomenon. In this paper, we focus on disorder motion that occurs in the crowded indoor publics, especially the narrow channel and safety exits and other special arteries. We put forward the improved social force model to depict pedestrians' behaviors, an orderly speed-stratification evacuation method to solve disorder problem, and shape-changed export to alleviate congestion. The result of this work shows an improvement of evacuation efficiency by $19.5 \%$. Guiding pedestrians' direction to slow down the influence of social forces has a guidance function in improving the efficiency of indoor emergency evacuation.
\end{abstract}

\section{INTRODUCTION}

Social force always exists in the evacuation process, the representatives include friction, collision, attraction and repulsion, a considerable number of scholars describe the evacuation model from these forces.

Francesco Farina proposed a social force model that was enriched with Laumond's human movement model, which presents the best features inherited from the two models. It can reliably reproduce human behavior in free space and highly congested environments, and automatically adapt to external shock without having to switch model.

Few studies have focused on the evacuation of multi-floor classroom buildings in a primary school, so Li proposed a stairunit model to describe the spatial topology of twisting stairwells and to describe the spatial relationship between stairwells and floors. Based on the stair-unit model, a schedule-line model was proposed to calculate evacuation paths in stair-units; a modified algorithm to calculate pedestrian forces were proposed to describe the evacuee movements in stairwells; and a projection strategy was proposed to model the 3-dimensional evacuation process in multi-floor buildings.

Colombi A present a microscopic-discrete mathematical model describing crowd dynamics in no panic conditions. Relevant improvements of our mathematical model with respect to similar microscopic-discrete approaches present in the literature are: (i) each pedestrian has his/her own dynamic gazing direction, which is regarded to as an independent degree of freedom and (ii) each walker is allowed to take dynamic strategic decisions according to his/her environmental awareness (new information acquired on the surrounding space through their visual region).

In addition to the refinement of the model, there are scholars studied on typical phenomenon.
Suzuno shows arch by social force model and image processing. The influence from obstacle to arch structure was discussed, and

it is suggested that these effects can lead to arch damage and enhance pedestrian flow at the exit. Matsuoka Takashi studied the location and size of obstructions and suggested that establish correct barriers for exit could reduce pedestrian's departure time.

Current regulations demand that at least two exits should be available for a safe evacuation during a panic situation. However, rooms having contiguous doors not always reduce the leaving time as expected. No attention has been given to this issue in the literature before Sticco I M did the work. They investigated the relation between the door's separation and the evacuation performance, and found that there exists a separation distance range that does not really improve the evacuation time, or it can even worsen the process performance. They report how the pedestrian's dynamics differ when the separation distance between two exit doors changes and how this affects the overall performance.

Our study focused on the influence of social force on personnel and trying to alleviate congestion. Against disorder, speed stratification is proposed inspired by the lane. Let pedestrians evacuate on lane grouped by different speed, and the social force between pedestrians can be reduced. It can have a great improvement of efficiency.

\section{SOCIAL FORCE MODEL}

We studied evacuation under single-channel with one exit case. Assuming that collision will occur during movement because of speed difference, in which process produced social force including friction, collision, attraction and repulsion. Friction and collision will reduce pedestrian's speed or cause varying degrees 
of damage to pedestrians; attraction and repulsion were thought of psychological factors. We have

$$
m_{i} \frac{d v_{i}}{d t}=m_{i} \frac{v_{i}^{0}(t) \boldsymbol{e}_{i}^{0}(t)-\boldsymbol{v}_{i}(t)}{\tau_{i}}+\sum_{j(\neq i)} \boldsymbol{f}_{i j}+\sum_{W} \boldsymbol{f}_{i W}
$$

Where the position of $\boldsymbol{r}_{i}$ is related with speed $\boldsymbol{v}_{i}(t)=d \boldsymbol{r}_{i} / d t$; $d_{i j}=\left\|\boldsymbol{r}_{i}-\boldsymbol{r}_{j}\right\|$ is the centroid distance between pedestrian $i, j$; $\boldsymbol{n}_{i j}=\left(n_{i j}^{1}, n_{i j}^{2}\right)=\left(\boldsymbol{r}_{i}-\boldsymbol{r}_{j}\right) / d_{i j}$ is a vector point from $j$ to $i$. When $d_{i j}<r_{i j}=\left(r_{i}+r_{j}\right)$, there is a contact between pedestrians.

Social force between pedestrians:

$$
\begin{gathered}
\boldsymbol{f}_{i j}=k g\left(r_{i j}-d_{i j}\right) \boldsymbol{n}_{i j}+k g\left(r_{i j}-d_{i j}\right) \Delta v_{j i}^{t} \boldsymbol{t}_{i j}+ \\
\boldsymbol{\nabla}_{d_{i j}}\left(\ln {d_{i j}}^{2}+\frac{D_{i j}{ }^{2}}{d_{i j}{ }^{2}}\right)
\end{gathered}
$$

$k\left(r_{i j}-d_{i j}\right) \boldsymbol{n}_{i j}$ represents the collision. $k\left(r_{i j}-d_{i j}\right) \Delta v_{j i}^{t} \boldsymbol{t}_{i j}$ is the friction, where $\boldsymbol{t}_{i j}=\left(-n_{i j}^{2}, n_{i j}^{1}\right)$ is in tangential direction, and $\Delta v_{j i}^{t}=\left(\boldsymbol{v}_{j}-\boldsymbol{v}_{i}\right) \boldsymbol{t}_{i j}$ is the tangential speed difference. $\boldsymbol{\nabla}_{d_{i j}}\left(\ln d_{i j}{ }^{2}+\frac{D_{i j}{ }^{2}}{d_{i j}{ }^{2}}\right)$ represents attraction and repulsion, $D_{i j}{ }^{2}$ is the threshold for centroid distance, which shows attraction when $d_{i j}>D_{i j}{ }^{2}$ and repulsion when $d_{i j}<D_{i j}{ }^{2}$.

Social force between pedestrians and walls are similar to that between pedestrians. $d_{i W}$ is the distance between pedestrian and wall; $\boldsymbol{n}_{i W}$ is the perpendicular direction from pedestrian to wall; $\boldsymbol{t}_{i W}$ is the tangential direction, which parallel to the wall.

$$
\begin{gathered}
\boldsymbol{f}_{i W}=k g\left(r_{i}-d_{i W}\right) \boldsymbol{n}_{i W}-k g\left(r_{i}-d_{i W}\right)\left(\boldsymbol{v}_{i} \boldsymbol{t}_{i W}\right) \boldsymbol{t}_{i W}+ \\
\boldsymbol{\nabla}_{d_{i W}}\left(\ln d_{i W}{ }^{2}+\frac{D_{i W}{ }^{2}}{d_{i W}{ }^{2}}\right)
\end{gathered}
$$

Social force is related with $d_{i j}$. The greater the population density, the smaller the $d_{i j}$. We concluded:1, when $d_{i j}>r_{i j}$, the attraction and repulsive force will dominant movement direction, and trigger disorder problems; 2, when $d_{i j}<r_{i j}$, friction and collision take domination, pedestrians keep colliding with others (when the crowd density is large) like a perturbation and will delay the evacuation time; 3 , when the crowd density is too large, the faster-is-slower shows up: pedestrians cannot move as they want due to friction, which bind the crowd together and make their speed slow down. This will cause arch-like blocking when happened nearby door. The dominated social forces under different crowd densities are summarized:

$$
\rho\left(\text { per } / m^{2}\right)\left\{\begin{array}{l}
>4.2, \text { friction } \\
>0.75, \text { repulsion } \\
=0.75, \text { balabced state } \\
<0.75, \text { attraction }
\end{array}\right.
$$

When $d_{i j}<r_{i j}$, pedestrians suffer smaller social force as $d_{i j}$ get larger. We propose a method to alleviate social force and disorder problem by 'reducing' crowd density. It should improve evacuation efficiency.

An object will be deformed when suffering external force and expand toward force direction. Similarly, the crowd will be deformed in single-channel evacuation process due to attraction, and the front is normal distribution. Speed stratification is used to conform to the movement tendency to improve efficiency.

We consider to rearrange pedestrian's position by speed. The middle part evacuates fast and will separate with other parts, leading to reduction of local population of local population density and reshaping the moving direction. The ladder-like distribution minimums pedestrians' speed difference $\Delta v_{j i}^{t}=$ $\left(\boldsymbol{v}_{j}-\boldsymbol{v}_{i}\right) \boldsymbol{t}_{i j}$ lead to friction reducing correspondingly.

Taking middle part as a group, we divided the crowd into three groups and idealized: 1 , in evacuation process, pedestrians in group have the same speed and the group structure will be stable, only boundary where exists speed difference will produce external force; 2 , group direction is limited by space to point at the export that solved the disorder problem.

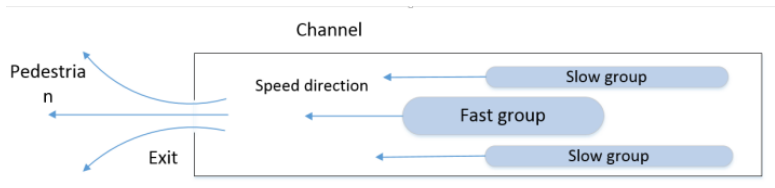

Figure 1. Speed Stratification

There is another problem in the single-channel evacuation process: bottlenecks. Helbing studied this problem and had a result: obvious bottleneck makes great blocking. That is, the smaller the angle of the bottleneck $\alpha$, the lighter the blocking situation. We considered to reduce $\alpha$ which can be conducive to reduce the population density at the exit and the social force as a consequence. We have simulation to verify this idea.

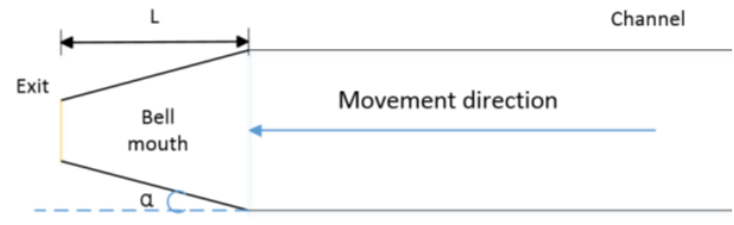

Figure 2. Trapezoidal Export

\section{SIMULATION AND DISSCUSSION}

\subsection{Speed separation}

We simulated $40 \mathrm{~m}$ long, $6 \mathrm{~m}$ wide single corridor. The initial conditions are the same, we got simulation results: speedseparation for $40.8 \mathrm{~s}$, no separation $48.8 \mathrm{~s}$, efficiency of stratification improved by $16.4 \%$. During the no separation process, we observed disorder phenomenon, which increased evacuate route and make pedestrians accumulated. Faster-isslower often occur, we can saw herd clearly which slow down the evacuation efficiency. On the country, speed-separation avoid friction and disorder. We can saw straight travel route clearly, blocking at the exit reduced by nearly two-thirds. Speedseparation has better evacuation effect.

$$
\text { Exited: } 0 / 656
$$
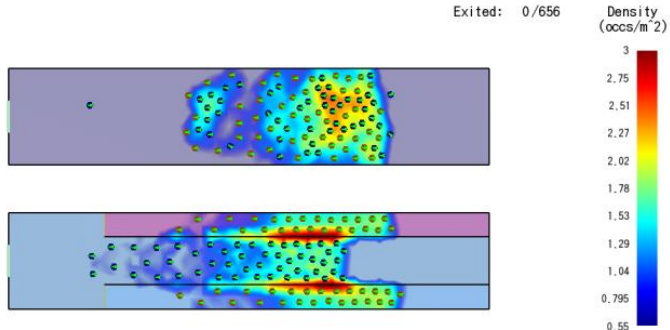

9.7

Figure 3. Comparison of stratification and no-stratification

We also compared the regional width of velocity stratification. Under the same condition, we set different width of speed area and got almost the same evacuation time around $41 \mathrm{~s}$.

Nonetheless, we found that the narrower the middle area the 
later the crowd started, in the simulation. As the rear pedestrian has no movement space, they have to wait for the front pedestrians move away. This caused the pedestrians accumulation in the start place. The front row of pedestrians' stagnation time is zero, and the next rows has an iteration in stagnation time, and to last row it delayed about $4 \mathrm{~s}$. Although this phenomenon has little effect on the total evacuation time in the simulation, we should take the psychological factors of pedestrians in reality evacuation into account and set the width of the middle area in the corridor one-half and above, because large crowd density tends to cause accident.

\subsection{Export shape improvement}

We simulated single-channel corridor with a trapezoidal export length $40 \mathrm{~m}$, width $6 \mathrm{~m}$, simulation staff 100 . Changing the angle $\alpha$, and we can get the evacuation time tendency as in figure 4 :

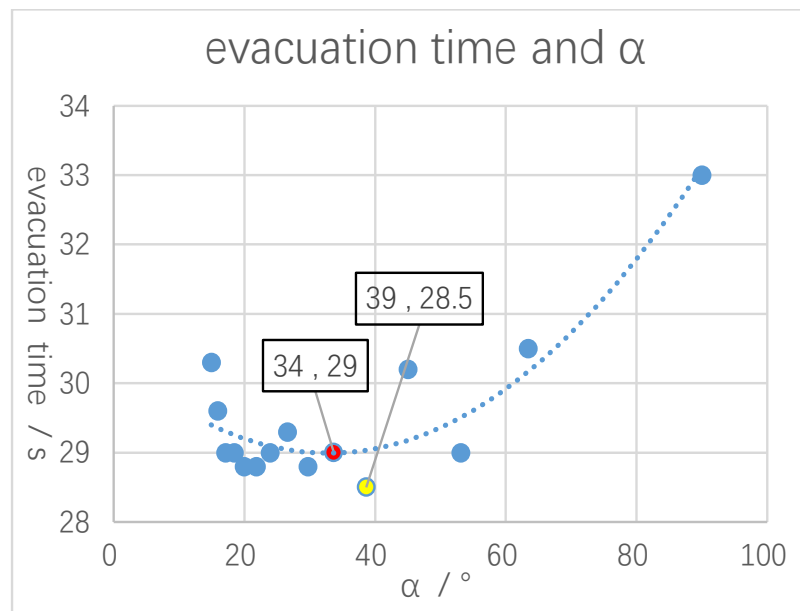

Figure 4. Evacuation time change with angle $\alpha$

The red dot is the lowest point of the tendency, due to the $0^{\circ}$ exit shape infinitely close to $90^{\circ}$. It should have the shortest evacuation time, around $29 \mathrm{~s}$ while $\alpha=34^{\circ}$. Yellow point is the actual shortest evacuation time, around $28.5 \mathrm{~s}$ while $\alpha=39^{\circ}$. When $\alpha=90^{\circ}$ or $\alpha=0^{\circ}$, that is, when the export is normal, the evacuation time is $33 \mathrm{~s}$. We got that evacuation time improved by about $14 \%$ while the export shape is improved.

While building export, we found that $\alpha$ changes as length changes. The main factor of evacuation time is not clear, so we used quantitative analysis method to measure weights:

1) changing the shape of the trapezoidal export, while ensuring $\alpha=34^{\circ}$, the evacuation time:

\begin{tabular}{|c|c|c|c|}
\hline Length & $0.75 \mathrm{~m}$ & $1.5 \mathrm{~m}$ & $3 \mathrm{~m}$ \\
\hline Evacuation time & $32.5 \mathrm{~s}$ & $31.8 \mathrm{~s}$ & $30.8 \mathrm{~s}$ \\
\hline
\end{tabular}

Table 1. Change trapezoidal export while keep angle quantitative
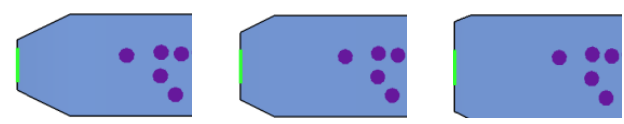

Figure 5. Change trapezoidal export while keep angle quantitative

2) changing the shape of the trapezoidal export, while ensuring $\mathrm{L}=3 \mathrm{~m}$, the evacuation time:

\begin{tabular}{|c|c|c|c|}
\hline$\alpha$ & $27^{\circ}$ & $18^{\circ}$ & $9^{\circ}$ \\
\hline Evacuation time & $35 \mathrm{~s}$ & $33.8 \mathrm{~s}$ & $33 \mathrm{~s}$ \\
\hline
\end{tabular}

quantitative
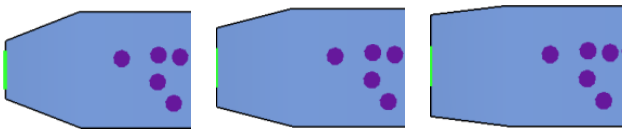

Figure 6. Change trapezoidal export while keep length quantitative

It can be seen that whether changing the angle or length, the evacuation efficiency is not as good as the original simulation, and some even longer than normal export shape evacuation time. The reason is that while the width of the outlet is certain, there is a certain width of wall left around the exit and block pedestrians, which led to the accumulation of pedestrians. And also because of narrow trapezoid area, the density of pedestrians is larger than normal condition, friction-dominated faster-isslower phenomenon makes the crowd slow down, so did the evacuation efficiency.

In view of the export width problem, we have done simulation that export width changes as the trapezoid changed:

3) Maintain $\alpha=34^{\circ}$ unchanged, change the length, the evacuation time:

\begin{tabular}{|l|l|l|l|l|}
\hline Length & $3 \mathrm{~m}$ & $2.25 \mathrm{~m}$ & $1.5 \mathrm{~m}$ & $0.75 \mathrm{~m}$ \\
\hline Export width & $2 \mathrm{~m}$ & $3 \mathrm{~m}$ & $4 \mathrm{~m}$ & $5 \mathrm{~m}$ \\
\hline Evacuation time & $29 \mathrm{~s}$ & $24.5 \mathrm{~s}$ & $24 \mathrm{~s}$ & $24 \mathrm{~s}$ \\
\hline
\end{tabular}

Table 3. Change export width as length changed

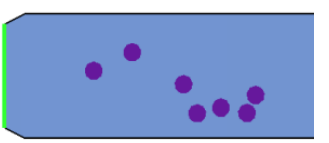

Figure 7. Change export width as length changed

4) Maintain length $3 \mathrm{~m}$ unchanged, change the angle, the evacuation time:

\begin{tabular}{|c|c|c|c|c|}
\hline Angle & $34^{\circ}$ & $27^{\circ}$ & $18^{\circ}$ & $9^{\circ}$ \\
\hline Export width & $2 \mathrm{~m}$ & $3 \mathrm{~m}$ & $4 \mathrm{~m}$ & $5 \mathrm{~m}$ \\
\hline Evacuation time & $29 \mathrm{~s}$ & $25.3 \mathrm{~s}$ & $24 \mathrm{~s}$ & $24 \mathrm{~s}$ \\
\hline
\end{tabular}

Table 4. Change export width as $\alpha$ changed

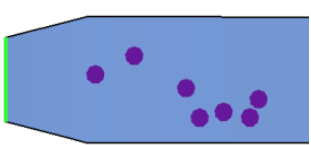

Figure 8 . Change export width as $\alpha$ changed

From the two simulations, it can be concluded that when the export width is $3 \mathrm{~m}$, it will not be blocked. Increasing width will not save evacuation time. Take construction cost into account, $2 \mathrm{~m}$ is enough for real evacuate.

We combined the trapezoidal export with speed-stratification and got evacuation time $40.1 \mathrm{~s}$ while $\alpha=34^{\circ}$. Under the same 
conditions to simulate speed-stratification, trapezoidal export and normal case, the results are $42.1 \mathrm{~s}, 43.9 \mathrm{~s}$ and $49.8 \mathrm{~s}$.

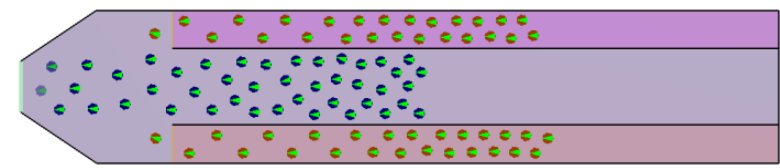

Figure 9. Trapezoidal export and speed-separation

It can be seen that in crowded public places, especially narrow channel and safe exit, the speed-stratification and trapezoidal export should slow down the impact of social forces, clogging and trampling. It plays a positive guiding role to improve the efficiency of indoor emergency evacuation.

\section{SUMMARY}

We analyze the social force of pedestrians, and think that friction and collision are external force that will reduce velocity during movement; attraction and repulsion are psychological factors, that will make people lost direction, and resulting in disordered evacuation. Attraction and repulsion are the representation of psychological factors, while other psychological factors that affect lightly are ignored in this article.

We have done the following research in single-channel: 1 , social force between pedestrians and its influence on evacuation time; 2 , speed-separation and its influence; 3 , trapezoidal export and its influence; 4, export width and its influence. It shows that the export threshold should be $2 \mathrm{~m}$. The speed-separation can help improve efficiency by about $16 \%$ and mitigate disorder. The trapezoidal export can help improve efficiency by about $14 \%$ and ease congestion. The cooperation will improve $19.5 \%$. The result shows a great improvement of efficiency on crowded corridor and could be applied in reality by painting different colors of speed area on floor for people to recognize.

\section{REFERENCES}

Farina F, Fontanelli D, Garulli A, et al, 2016. When Helbing meets Laumond: The Headed Social Force Model. IEEE Decision and Control, 3548-3553.

Li W, Li Y, Yu P, et al, 2016. Modeling, simulation and analysis of the evacuation process on stairs in a multi-floor classroom building of a primary school. Physica A Statistical Mechanics \& Its Applications, 469:157-172.

Colombi A, Scianna M, Alaia A, 2016. A discrete mathematical model for the dynamics of a crowd of gazing pedestrians with and without an evolving environmental awareness. Computational \& Applied Mathematics, 1-29.

Han Y, Liu H, 2016. Modified social force model based on information transmission toward crowd evacuation simulation. Physica A Statistical Mechanics \& Its Applications, 469.

Gao Y, Chen T, Luh P B, et al, 2016. Modified Social Force Model Based on Predictive Collision Avoidance Considering Degree of Competitiveness. Fire Technology, 1-21.

Del Sent A, Roisenberg M, 2015. Fuzzy Social Force Model. IEEE International Conference on Fuzzy Systems, 1-8.

Suzuno K, Tomoeda A, Iwamoto M, et al, 2015. Dynamic Structure in Pedestrian Evacuation: Image Processing
Approach. Traffic and Granular Flow '13, Springer International Publishing, 195-201.

Sticco I M, Frank G A, Cerrotta S, et al, 2016. Room evacuation through two contiguous exits. Physica A Statistical Mechanics \& Its Applications, 474:172-185.

Zhao Y, Li M, Lu X, et al, 2017. Optimal layout design of obstacles for panic evacuation using differential evolution. Physica A Statistical Mechanics \& Its Applications, 465:175194

Sylvain Faure, Bertrand Maury, 2014. Crowd motion from the granular standpoint. Mathematical Models \& Methods in Applied Sciences, 25(03):463-493.

Parisi D R, Soria S A, Josens R, 2014. Faster-is-slower effect in escaping ants revisited: Ants do not behave like humans. Safety Science, 72:274-282.

Matsuoka T, Tomoeda A, Iwamoto M, et al, 2015. Effects of an Obstacle Position for Pedestrian Evacuation: SF Model Approach. Traffic and Granular Flow '13, Springer International Publishing, 163-170. 